\title{
Temperature Dependence of Antiferromagnetic Interlayer Exchange Coupling in EuS-PbS Multilayers
}

\author{
M. Chernyshova ${ }^{a}$, L. KowalczyK ${ }^{a}$, M. Baran ${ }^{a}$,

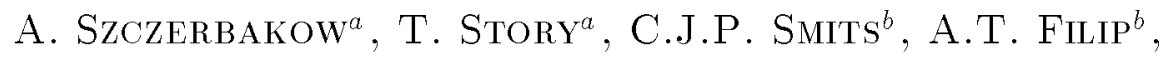 \\ H.J.M. SwaGTEN ${ }^{b}$, W.J.M. DE JONGE ${ }^{b}$ AND A.Yu. SiPATOV ${ }^{c}$ \\ ${ }^{a}$ Institute of Physics, Polish Academy of Sciences \\ al. Lotników 32/46, 02-668 Warsaw, Poland \\ ${ }^{b}$ Eindhoven University of Technology \\ P.O. Box 513, 5600 MB Eindhoven, The Netherlands \\ ${ }^{c}$ National Technical University KPI, 21 Frunze Str., 61002 Kharkov, Ukraine
}

\begin{abstract}
Antiferromagnetic interlayer exchange coupling in semiconductor EuS-PbS-EuS ferromagnetic trilayers grown on PbS (001) substrates with ultrathin $(0.6-1.2 \mathrm{~nm})$ nonmagnetic PbS spacers is studied by SQUID magnetometry and model calculations. Analysis of the experimentally observed magnetic field and temperature dependence of the magnetization of EuS-PbS structures reveals a rapid decrease in the interlayer coupling energy with increasing temperature indicating a temperature dependence of the microscopic coupling mechanism acting in these all-semiconductor ferromagnetic/ nonmagnetic multilayers.
\end{abstract}

PACS numbers: $75.20 . \mathrm{Ck}, 75.30$.Et

\section{Introduction}

EuS-PbS multilayers (superlattices or trilayers) are all-semiconductor ferromagnetic/nonmagnetic heterostructures exhibiting a ferromagnetic transition at about $T_{\mathrm{c}}=17 \mathrm{~K}$ (in structures with thick EuS layers). In EuS-PbS multilayers with ultrathin (below about $3 \mathrm{~nm}$ ) EuS layers the ferromagnetic Curie temperature is reduced down to about $2 / 3$ of its value for the bulk crystals but the transition 
is observed even in 2 monolayer $(0.6 \mathrm{~nm})$ thick layers of EuS. Magnetic properties of EuS-PbS multilayers are also influenced by strain, present in these structures mainly due to the difference in thermal expansion coefficients of the multilayer and the $\mathrm{KCl}$ or $\mathrm{BaF}_{2}$ substrates $[1,2]$. The effect is particularly large for structures grown on $\mathrm{KCl}$ substrates, in which strong compressive in-plane strain results in about $3 \mathrm{~K}$ increase in the Curie temperature. In EuS-PbS heterostructures the ferromagnetic layers of EuS (constituting model Heisenberg nonmetallic ferromagnet) are separated by very well lattice-matched diamagnetic layers of $\mathrm{PbS}$ (well-known IV-VI semiconductor compound). Both these materials crystallize in the rock salt crystal structure with lattice parameters $0.597 \mathrm{~nm}$ and $0.594 \mathrm{~nm}$ for EuS and $\mathrm{PbS}$, respectively, and are known to form pseudomorphic heterostructures up to the total thickness of about $200 \mathrm{~nm}$. It was observed that in EuS-PbS semiconductor multilayers with ultrathin (about $1 \mathrm{~nm}$ ) diamagnetic spacer layers the ferromagnetic layers of EuS are coupled antiferromagnetically via the nonmagnetic $\mathrm{PbS}$ spacer layer. This effect is observed for EuS-PbS superlattices and trilayer structures grown along the [001] direction both on $\mathrm{KCl}$ (neutron reflectivity [3] and magnetization [3-6] studies) and $\mathrm{PbS}$ (magnetization study [4-6]) substrates. It was also found for closely related EuS-YbSe superlattices grown on $\mathrm{KCl}$ (neutron reflectivity study $[7,8]$ ) but not observed for EuS-PbS structures grown on $\mathrm{BaF}_{2}$ substrates with [111] growth direction.

In this work, we present the experimental evidences (based on magnetization measurements) and discuss the physical origin of the temperature dependence of the antiferromagnetic interlayer exchange energy in EuS-PbS-EuS structures grown on PbS (001) substrates. In contrast to metallic ferromagnetic multilayers, for which the interlayer coupling is usually studied at temperatures much lower than the Curie temperature, we exploit here the possibility offered by the EuS based ferromagnetic systems and analyze the interlayer coupling in the entire relevant temperature range $\left(0.1 \leq T / T_{\mathrm{c}} \leq 1\right)$

\section{Magnetic measurements}

The temperature and magnetic field dependence of magnetization was studied in $\operatorname{EuS}(t)-\operatorname{PbS}(d)-\operatorname{EuS}(t)$ trilayers $(t=3-20 \mathrm{~nm} ; d=0.6-6 \mathrm{~nm})$ grown by a high vacuum deposition on freshly cleaved $\mathrm{PbS}(001)$ monocrystalline substrates. Due to the extremely high melting point and very low vapor pressure of EuS, an electron gun has to be employed for the evaporation of this material. For the evaporation of $\mathrm{PbS}$, a standard resistively heated $W$ boat was used. In all the investigated structures there also is a $\mathrm{PbS}$ buffer layer (about $100 \mathrm{~nm}$ thick) as well as about $50 \mathrm{~nm}$ thick $\mathrm{PbS}$ capping layer for preventing from oxidation. Although two different $\mathrm{PbS}$ source materials were used for evaporation (stoichiometry controlled, $n$ and $p$-type polycrystals), all the PbS layers grown in high vacuum at substrate temperature of about $350^{\circ} \mathrm{C}$ revealed $n$-type conductivity. 
In agreement with our recent experimental study of the ferromagnetic transition in EuS-PbS multilayers grown on PbS substrates [9], our structures show the transition at about $16 \mathrm{~K}$. As in EuS-PbS multilayers one observes a very strong shape anisotropy with demagnetization field $4 \pi M$ of almost $1.5 \mathrm{~T}$ (at $T=2 \mathrm{~K}$ ), the layers are magnetized in the plane of the structure. Therefore, our measurements of hysteresis loops were carried out applying an external magnetic field in the (001) plane of the layer along both hard [100] and easy [110] magnetization axis as established from magnetization measurements of uncoupled EuS-PbS heterostructures on PbS substrate [6]. The magnetization measurements were carried out by a superconducting quantum interference device (SQUID) magnetometer in the temperature range of $2-25 \mathrm{~K}$ and magnetic field range up to $300 \mathrm{Oe}$, i.e. covering the entire magnetic hysteresis range of magnetic fields and both paramagnetic and ferromagnetic range of temperatures.

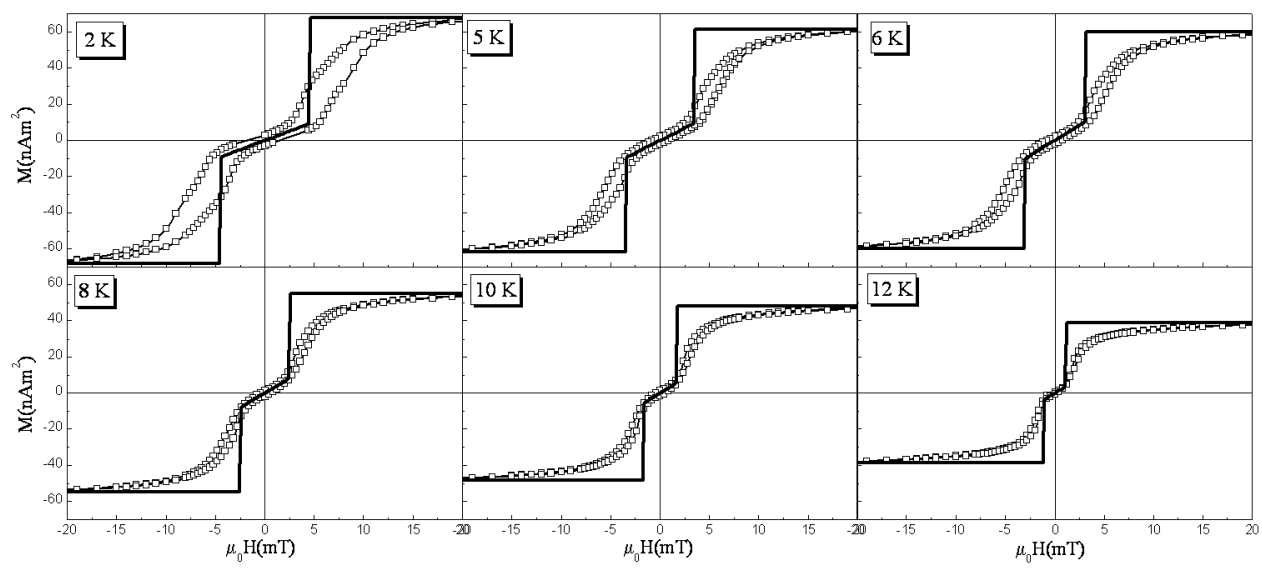

Fig. 1. Magnetic hysteresis loops (at temperatures shown in the figure) of $\operatorname{EuS}(3 \mathrm{~nm})-\mathrm{PbS}(0.75 \mathrm{~nm})-\mathrm{EuS}(3 \mathrm{~nm})$ trilayer grown on $\mathrm{PbS}(001)$ substrate with $100 \mathrm{~nm} \mathrm{PbS}$ buffer and $50 \mathrm{~nm} \mathrm{PbS}$ cap layer. An external magnetic field was applied in the plane of the multilayer along [110] crystal direction (easy axis of magnetization). Open symbols present experimental data whereas solid lines show model calculations.

Magnetic hysteresis loops of $\operatorname{EuS}(3 \mathrm{~nm})-\mathrm{PbS}(0.75 \mathrm{~nm})-\mathrm{EuS}(3 \mathrm{~nm}) / \mathrm{PbS}(001)$ trilayer exhibiting a relatively strong antiferromagnetic interlayer coupling are presented (at various temperatures) in Fig. 1. In contrast to regular, approximately square-like, loops found in noncoupled trilayers (with $\mathrm{PbS}$ spacer thicker than about $2 \mathrm{~nm}$ ) [1, 2, 6], the experimental data clearly show a characteristic almost zero magnetic remanence and a low field linear "plateau" with a well-defined external magnetic field corresponding to the switch between antiferromagnetic- and ferromagnetic-like alignment of magnetization vectors of two EuS layers. Qualitatively, the magnetization loops of exchange-coupled EuS-PbS/PbS (001) mul- 
tilayers are very similar as for the previously studied EuS-PbS structures grown on $\mathrm{KCl}(001)$ substrates [1, 2]. The key experimental observation illustrated in Fig. 1 is that the above-mentioned experimental features related to antiferromagnetic interlayer coupling exhibit a strong temperature dependence, which will be quantitatively analyzed in the next section.

\section{Discussion and conclusions}

To quantitatively analyze the experimental observations, the $M(H)$ dependence was modeled theoretically by determining the component of the total magnetization of the trilayer along the applied magnetic field, i.e. the physical quantity measured by a SQUID magnetometer. It depends, in particular, on the mutual orientation of the magnetization vectors $\boldsymbol{M}_{1}(T)$ and $\boldsymbol{M}_{2}(T)$ of the two EuS layers that corresponds to the minimum of the total magnetic energy of the system. In the modeling, one considers two exchange coupled ferromagnetic layers of thicknesses $t_{1}$ and $t_{2}$ with an area density of the interlayer exchange energy given by the standard expression: $E_{\mathrm{ex}}=I_{\mathrm{ex}} \boldsymbol{M}_{1} \boldsymbol{M}_{2} / \boldsymbol{M}_{1} \boldsymbol{M}_{2}=I_{\mathrm{ex}} \cos \phi$, where $\phi$ is the angle between vectors $\boldsymbol{M}_{1}$ and $\boldsymbol{M}_{2}$. Each individual magnetic layer is placed in an external magnetic field $\boldsymbol{H}$ applied in the plane of the multilayer and contributes a Zeeman energy (per unit area): $E_{\mathrm{Z}}=-\boldsymbol{M}_{1}(T) \boldsymbol{H} t_{1}-\boldsymbol{M}_{2}(T) \boldsymbol{H} t_{2}$. The individual layers exhibit also temperature dependent in-plane cubic (fourfold) magnetic anisotropy with an area density $E_{\mathrm{A}}=K_{1}(T) t_{1} \sin ^{2} \phi_{1} \cos ^{2} \phi_{1}+K_{2}(T) t_{2} \sin ^{2} \phi_{2} \cos ^{2} \phi_{2}$. Here, $\phi_{1}$ and $\phi_{2}$ are the angles between crystal axis [100] and magnetization vectors $M_{1}$ and $M_{2}$, respectively. In EuS-PbS/PbS (001) layers the anisotropy constants $K_{1,2}$ are negative and [110] and [1-10] are easy axes of magnetization. The angle $\phi(H, T)=\phi_{2}-\phi_{1}$ shows a characteristic temperature and magnetic field dependence [4]: for temperatures just below the Curie temperature $\phi=0$ (parallel orientation of both magnetization vectors) but it starts to increase at a well-defined temperature (lower for higher external magnetic field). In the model calculations the temperature dependence of the anisotropy constant was taken as $K(T) \propto M_{\mathrm{s}}^{4}(T)$, which is expected for the cubic ferromagnetic systems with a dominant single-ion type magnetocrystalline anisotropy. The results of the modeling are plotted in Fig. 1 (solid lines) for the case of magnetic field applied along an easy magnetization axis. The minimalization procedure applied corresponds to a global minimum approach and it cannot predict any hysteresis of $M(H)$ dependence. During the fitting procedure two experimental features were considered of primary importance for the determination of the exchange coupling energy: the slope of the linear part of $M(H)$ dependence at low magnetic fields and the switching field. The antiferromagnetic interlayer coupling is also known to influence the saturation field of hysteresis loop which can be used to evaluate the exchange energy. In the case of EuS-PbS multilayers substantial rounding of the $M(H)$ dependence at fields close to the saturation is also observed for uncoupled layers. Therefore, in exchange coupled EuS-PbS-EuS trilayers the $M(H)$ dependence (close to the saturation) 
is influenced by both magnetization processes within individual EuS layers and antiferromagnetic interlayer coupling between them $[1,2,6,8]$. It makes this part of magnetic hysteresis loop not suitable for determination of the coupling energy.
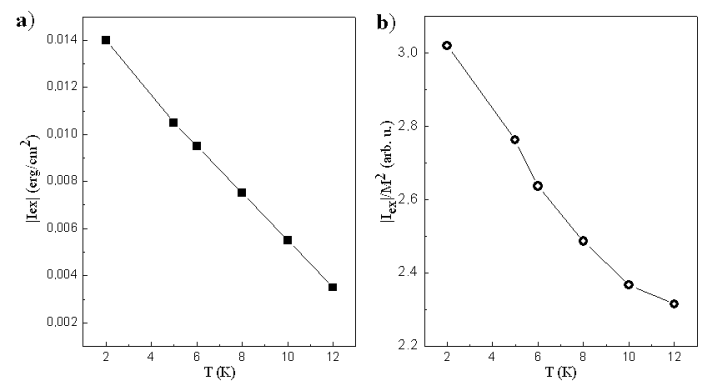

Fig. 2. (a) Temperature dependence of the antiferromagnetic exchange energy in Eu-PbS-EuS/PbS (001) trilayer as determined from the model analysis presented in Fig. 1. (b) The temperature dependence of the exchange integral parameter $J_{\text {ex }}=$ $I_{\mathrm{ex}} / M_{\mathrm{s}}^{2}(T)$ is presented for the same sample.

Figure 2 a presents the results of such a modeling of the experimental data, which reveals that the antiferromagnetic exchange energy $I_{\mathrm{ex}}(T)$ increases rapidly with decreasing temperature, i.e. increasing saturation magnetization $M_{\mathrm{s}}$ of each of the layers. In Fig. 2b the temperature dependence of the exchange integral parameter, defined as $J_{\mathrm{ex}} \propto I_{\mathrm{ex}} / M_{\mathrm{s}}^{2}(T)$, is presented showing that the strength of the very microscopic exchange mechanism is also increasing with decreasing temperature. Qualitatively, the temperature dependence of the exchange integral is $J_{\text {ex }} \propto M_{\mathrm{s}}(T)$, i.e., it follows the temperature dependence of the magnetization of the layer. This implies that the exchange energy depends on temperature as $I_{\mathrm{ex}} \propto M_{\mathrm{S}}^{3}(T)$. The analysis of a broader spectrum of EuS-PbS structures both on $\mathrm{KCl}$ and $\mathrm{PbS}$ substrates, discussed in Ref. [6], shows that even a more rapid temperature dependence of the exchange integral is also found: $J_{\mathrm{ex}} \propto M_{\mathrm{s}}^{\alpha}(\mathrm{T})$, with $\alpha=1-2$.

The experimentally established temperature dependence of the antiferromagnetic interlayer exchange in EuS- $\mathrm{PbS}$ mulilayers brings additional information vital for the critical evaluation of the physical models of the interlayer coupling in all-semiconductor multilayers. Due to the lack of the sufficient number of quasi-free charge carriers (in both ferromagnetic and nonmagnetic layers), in all-semiconductor magnetic heterostructures new mechanisms of interlayer exchange coupling are expected to be effective. In particular, the canonical Ruderman-Kittel-Kasuya-Yosida (RKKY) coupling via quasi-free electrons observed in metallic multilayers [10] is strongly modified in EuS-PbS structures due to the lack of electrons in ferromagnetic EuS layer and the two-dimensional character of electron gas in the PbS quantum well [11]. Particularly important 
for all-semiconductor magnetic multilayers are mechanisms requiring no free electrons but involving spin dependent transitions of electrons from the valence band. In the model of Blinowski and Kacman [12] the total spin dependent energy of valence electrons in semiconductor magnetic superlattice is calculated taking into account transitions in the entire Brillouin zone, whereas in the model of Dugaev et al. [13] the Bloembergen-Rowland-type coupling (involving only electronic transitions close to conduction and band edges) is analyzed. Taking into account the realistic (at the atomic scale) picture of the ultrathin ferromagnet-nonmagnet-ferromagnet heterostructure one has also to consider the dipolar interactions which, although negligible for an ideal layered system, may become important for structures with a small scale domain structure or a strong interface roughness [14].

The first, well-established, experimental finding that has to be explained by a successful theoretical model is the antiferromagnetic sign of the coupling observed for EuS-PbS and EuS-YbSe structures grown along [001] crystal direction. In fact, in the layer thickness and carrier concentration range relevant to our structures, only the Blinowski and Kacman (B-K) model and dipolar (domain structures related) interactions correctly explain this observation. These two mechanisms are expected to be responsible for the interlayer coupling in EuS-PbS multilayers in different regions of the spacer thickness: the B-K mechanism for ultrathin spacers below about $2 \mathrm{~nm}$ (relatively strong coupling, rapid exponential decay of the coupling with increasing spacer thickness, no dependence on the thickness of EuS layer) and the dipolar mechanism for thicker spacers (relatively weak coupling, slow decay with increasing spacer thickness, increase of the coupling for thicker EuS layers). This conclusion is supported by the observation of, predicted by the $\mathrm{B}-\mathrm{K}$ model, a $1 / 2^{t}$ decay of the coupling in EuS-PbS/KCl structures with ultrathin $(t \leq 1.2 \mathrm{~nm}$ ) PbS spacers (neutron studies [3]), and by the experimentally observed more rapid decay of the antiferromagnetic interlayer coupling with increasing thickness of the nonmagnetic spacer for EuS-based structures with wider band gap spacer material (neutron reflectivity comparative study of EuS-PbS and EuS-YbSe superlattices $[7,8,15])$. Our experimental results also suggest that the antiferromagnetic interlayer coupling in EuS-PbS structures with ultrathin $\mathrm{PbS}$ spacer layer is due to the mechanism of electronic ( $\mathrm{B}-\mathrm{K}$ model) and not magnetostatic (dipolar) origin. For the latter the standard $I_{\mathrm{ex}} \propto M_{\mathrm{s}}^{2}(T)$ dependence is expected whereas in the $\mathrm{B}-\mathrm{K}$ model the coupling is inherently related to the exchange splitting of the valence band, which, in the first approximation, is proportional to the magnetization of the EuS layer, resulting in an overall $I_{\mathrm{ex}} \approx M_{\mathrm{s}}^{2}(T)$ dependence. However, as finite temperature B-K model calculations are not available so far, this conclusion remains of qualitative character only.

In conclusion, the model analysis of the experimentally observed magnetic hysteresis loops in $\mathrm{EuS}-\mathrm{PbS}-\mathrm{EuS} / \mathrm{PbS}$ (001) trilayers indicates that apart from the expected dependence due to the temperature dependence of the magnetization of each of the individual magnetic layers also the multilayer microscopic exchange in- 
tegral $J_{\mathrm{ex}} \propto I_{\mathrm{ex}} / M_{\mathrm{s}}^{2}(T)$ is approximately proportional to the magnetization of the layers. This finding supports the models of interlayer coupling in all-semiconductor EuS-PbS and related structures as originating from the exchange splitting of the valence and conduction bands.

\section{Acknowledgments}

This work is supported in part by the State Committee for Scientific Research research projects No. 1 P03B05426 and No. PBZ-KBN-044/P03B/2001 as well as within European Community program ICA1-CT-2000-70018 (Center of Excellence CELDIS).

\section{References}

[1] A. Stachow-Wójcik, T. Story, W. Dobrowolski, M. Arciszewska, R.R. Gałązka, H.J.M. Swagten, W.J.M. de Jonge, A. Twardowski, A.Yu. Sipatov, Phys. Rev. B 60, 15220 (1999).

[2] T. Story, Phys. Status Solidi B 236, 310 (2003).

[3] H. Kępa, J. Kutner-Pielaszek, J. Blinowski, A. Twardowski, C.F. Majkrzak, T. Story, P. Kacman, R.R. Gałazka, K. Ha, H.J.M. Swagten, W.J.M. de Jonge, A.Yu. Sipatov, V.V. Volobuev, T.M. Giebultowicz, Europhys. Lett. 56, 54 (2001).

[4] M. Chernyshova, L. Kowalczyk, A. Szczerbakow, T. Story, C.J.P. Smits, H.J.M. Swagten, C.H.W. Swuste, J.K. Ha, W.J.M. de Jonge, A.Yu. Sipatov, V.V. Volobuev, J. Supercond. 16, 213 (2003).

[5] C.J.P. Smits, A.T. Filip, H.J.M. Swagten, W.J.M. de Jonge, M. Chernyshova, L. Kowalczyk, K. Grasza, A. Szczerbakow, T. Story, A.Yu. Sipatov, J. Appl. Phys. 95, 7169 (2004).

[6] C.J.P. Smits, A.T. Filip, H.J.M. Swagten, B. Koopmans, W.J.M. de Jonge, M. Chernyshova, L. Kowalczyk, K. Grasza, A. Szczerbakow, T. Story, W. Palosz, A.Yu. Sipatov, Phys. Rev. B 69, 224410 (2004).

[7] H. Kępa, P. Sankowski, P. Kacman, A.Yu. Sipatov, C.F. Majkrzak, T.M. Giebultowicz, J. Magn. Magn. Mater. 272-276, 323 (2004).

[8] P. Sankowski, H. Kępa, P. Kacman, A.Yu. Sipatov, C.F. Majkrzak, T.M. Giebultowicz, this conference.

[9] M. Chernyshova, E. Eusakowska, V. Domukhovski, K. Grasza, A. Szczerbakow, S. Wrotek, L. Kowalczyk, T. Story, C.J.P. Smits, H.J.M. Swagten, W.J.M. de Jonge, W. Palosz, A.Yu. Sipatov, V.V. Volobuev, Acta Phys. Pol. A 102, 609 (2002).

[10] P. Bruno, Acta Phys. Pol. A 91, 37 (1997).

[11] V.V. Zorchenko, A.Yu. Sipatov, V.V. Volobuev, Low Temp. Physics 29, 1208 (2003) (Russian edition).

[12] J. Blinowski, P. Kacman, Phys. Rev. B 64, 045302 (2001).

[13] V.K. Dugaev, V.I. Litvinov, W. Dobrowolski, T. Story, Solid State Commun. 110, 351 (1999).

[14] J.A. Borchers, P.M. Gehring, R.W. Erwin, J.F. Anker, C.F. Majkrzak, T.L. Hylton, K.R. Coffey, M.A. Parker, J.K. Howard, Phys. Rev. B 54, 9870 (1996).

[15] P. Sankowski, P. Kacman, Acta Phys. Pol. A 103, 621 (2003). 